\title{
The Derivation of Convergence Analysis for the Multistage Adomian Decomposition Method for solving the Autonomous Van der Pol system
}

\author{
Dr. Abbas Y. AL_Bayati \& Dr. Ann J. AL_Sawoor \\ \& \\ Merna A. Samarji \\ Department of Mathematics \\ College of Computers Sciences and Mathematics \\ Mousl University
}

Received

$10 / 01 / 2010$
Accepted

27 / 06 / 2010

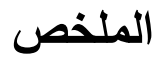

إن نظام Van der Pol قد نم حله باستخدام طريقة MADM [4]. حيث أثبتت هذه الطريقة كفاءتها ودقتها العالية في إيجاد الحلول العددية عند كل فترة زمنية مقارنة مع طريقة RK45 وطريقة ADM الكلاسيكية. في هذا البحث سنقوم باشتقاق تحليل التقارب لهذه الطريقة من خلال الاشتقاق العددي لطريقة (Homotopy Analysis) لهذا النظام ، سوف نبرهن بأنه $x_{i}(t=0)+\sum_{j=1}^{2} \sum_{n=0}^{\infty} a_{i j} \int_{0}^{t} x_{i n} d t+\sum_{n=1}^{\infty} x_{i n}(t) d t \quad$ إذا كانت المتسلسلة $x_{i n}(t)=\frac{\tilde{x}_{i 0}(t)}{n !}=\frac{\left.\frac{d^{n} \tilde{x}_{i}(t, \lambda)}{d \lambda^{n}}\right|_{\lambda=0}}{n !}=\left.\sum_{p=1}^{2} \sum_{q=1}^{2} a_{i p q} \int_{0}^{t} \frac{1}{n !} \frac{d^{n} \widetilde{\mathrm{R}}_{\mathrm{i} 2}\left(x_{p}, x_{q}, \lambda\right)}{d \lambda^{n}}\right|_{\lambda=0} d t, n \geq 1 \quad$ حي منقاربة وكانت هي الحل للنظام، فان المتتابعتين

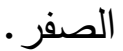

\footnotetext{
Abstract

The autonomous Van der Pol system was solved accurately by MADM [4]. The method has the advantage of giving the form of the numerical solution within each time interval which is not possible in purely numerical techniques like RK45 and classical ADM. In this paper we will derive the convergence of A Multistage Adomian Decomposition
} 
Method (MADM) by deriving the Homotopy Analysis Method for this system. We will show that if the series

$$
x_{i}(t=0)+\sum_{j=1}^{2} \sum_{n=0}^{\infty} a_{i j} \int_{0}^{t} x_{i n} d t+\sum_{n=1}^{\infty} x_{i n}(t) d t
$$

where

$$
x_{i n}(t)=\frac{\tilde{x}_{i 0}(t)}{n !}=\frac{\left.\frac{d^{n} \tilde{x}_{i}(t, \lambda)}{d \lambda^{n}}\right|_{\lambda=0}}{n !}=\left.\sum_{p=1}^{2} \sum_{q=1}^{2} a_{i p q} \int_{0}^{t} \frac{1}{n !} \frac{d^{n} \tilde{\mathrm{R}}_{\mathrm{i} 2}\left(x_{p}, x_{q}, \lambda\right)}{d \lambda^{n}}\right|_{\lambda=0} d t, \quad n \geq 1
$$

is convergent and it must be a solution of Van der Pol system, then the two sequences $\chi_{k}=\sum_{n=1}^{k} R_{i n}(t), v_{k}=\sum_{n=1}^{k} h_{i n}(t)$ are converges to zero.

\section{Introduction}

The study of nonlinear oscillators has been investigated in the development of the theory of dynamical systems. The Van der Pol oscillator (VPO), described by a second - order nonlinear differential equation which can be regarded as a description of a mass - spring damper system with a nonlinear position - dependent on damping coefficient or, equivalently, an RLC electrical circuit with a negative nonlinear resistor, such as electronics, biology or acoustics. It represents a nonlinear system with an interesting behavior that arises naturally in several applications.

This kind of nonlinear oscillator was used by Van der Pol in the 1920s to study oscillations in vacuum tube circuits.

In standard form, it is given by a second - order nonlinear differential equation of type:

$\ddot{x}+\mu\left(x^{2}-1\right) \dot{x}+x=0$,

Which can be reduced to two dimensional system of first order differential equations

$$
\begin{aligned}
& \dot{x}=y \\
& \dot{y}=-x-\mu\left(x^{2}-1\right) y
\end{aligned}
$$

Where $\mu(\mu>0)$ is a control parameter that reflects the degree of nonlinearity of the system. In studying the case $(\mu>>1)$, Van der Pol discovered the importance of what has been become known as relaxation oscillations [21].

The Multistage Adomian Decomposition Method (MADM) that will be handeled in this paper, has been applied to such as the multispecies Lotka-Volterra equations [16,17], the extended Lorenz system [20], the 
prey- predator problem [7] the chaotic Lorenz system [1,8-10], systems of ODEs [18], the classical Chen system $[15,1]$ and the Haldane equation for substrate inhibition enzyme kinetics [19]. The Homotopy Analysis Method (HAM), which was first introduced by Liao (see [11-14] and the references therein), is another technique used to derive an analytic solution for nonlinear operators. It consists of introducing embedding operators and embedding parameters where the solution is assumed to depend continuously on these parameters. The method has been used intensively by many authors and proved to be very effective in deriving an analytic solution of nonlinear differential equations [5,6,11,12]. In [4] we have been considered the (MADM) for solving Van der Pol system and the numerical results of this method show the efficient and powerful of the method as it compared with the classical Rungge - Kutta order four (RK45) and the classical Adomian Decomposition Method (ADM) for finding the solution of this system. In this paper we are interested to obtain the convergence of the Multistage Adomian Decomposition Method (MADM) by deriving the Homotopy Analysis Method (HAM) for this system.

\section{The Multistage Adomian Decomposition Method for solving system (2) \\ Recall [4], we consider the general system:}

$x_{i}^{\prime}=\sum_{j=1}^{2} a_{i j} x_{j}+\sum_{p=1}^{2} \sum_{q=1}^{2} a_{i p q} x_{p}^{2} x_{q} \quad, i=1,2$

Where $x_{1}^{\prime}=x^{\prime}(t)=\dot{x}$ and $x_{2}^{\prime}=y^{\prime}(t)=\dot{y}$, the prime denotes differentiation with respect to time.

If we denote the linear term (the first term on the r.h.s.) as $R_{i 1}$ and the nonlinear term (the second term) as $R_{i 2}$, then we can write the above system of equation in an operator form:

$$
L x_{i}=R_{i 1}+R_{i 2} \quad, \quad i=1,2
$$

Where $L$ is the differential operator $d(.) / d t$. Applying the inverse (integral) operator $L^{-1}$ to (4) we obtain

$$
x_{i}(t)=x_{i}(t=0)+L^{-1} R_{i 1}+L^{-1} R_{i 2} \quad, \quad i=1,2
$$

According to the $\operatorname{ADM}[2,3]$, the solution $x_{i}(t)$ will be given by the series

$$
x_{i}(t)=\sum_{n=0}^{\infty} x_{i n}(t) \quad, i=1,2
$$

Bearing this in mind, the linear term $R_{i 1}$ then becomes

$R_{i 1}=\sum_{j=1}^{2} \sum_{n=0}^{\infty} a_{i j} x_{i n}$,

so that $L^{-1} R_{i 1}$ will be given by: 


$$
L^{-1} R_{i 1}=\sum_{j=1}^{2} \sum_{n=0}^{\infty} a_{i j} \int_{0}^{t} x_{i n} d t \quad, i=1,2
$$

The non-linear term $R_{i 2}$ is decomposed as follows:

$R_{i 2}=\sum_{p=1}^{2} \sum_{q=1}^{2} a_{i p q} \sum_{n=0}^{\infty} A_{i n, p, q}$,

Where the $A_{i n, p, q}$ 's are the so-called Adomian polynomials, computed by using Algorithm [4] or using the following formula:

$A_{i n, p, q}=\frac{1}{n !} \frac{d^{n}}{d \lambda^{n}}\left[M\left(\sum_{k=0}^{\infty} \lambda^{k} x_{k p}, \sum_{k=0}^{\infty} \lambda^{k} x_{k q}\right)\right]_{\lambda=0}$

Where $M(x, y)=x^{2} y$ for each $n=0,1,2, \ldots$ Moreover, $L^{-1} R_{i 2}$ will be given by

$$
L^{-1} R_{i 2}=\sum_{p=1}^{2} \sum_{q=1}^{2} a_{i i p q} \sum_{n=0}^{\infty} \int_{0}^{t} A_{i n, p, q} d t
$$

Putting (6), (8), (9) into (5) we then have for each $i=1,2$

$$
\sum_{n=0}^{\infty} x_{i n}(t)=x_{i}(t=0)+\sum_{j=1}^{2} \sum_{n=0}^{\infty} a_{i j} \int_{0}^{t} x_{i n} d t+\sum_{p=1}^{2} \sum_{q=1}^{2} a_{i p q} \sum_{n=0}^{\infty} \int_{0}^{t} A_{i n, p, q} d t \text {, }
$$

Consequently, for each $i=1,2$ we have

$$
\begin{aligned}
& x_{i 0}=x_{i}(t=0), \\
& x_{i 1}=\sum_{j=1}^{2} a_{i j} \int_{0}^{t} x_{i 0} d t+\sum_{p=1}^{2} \sum_{q=1}^{2} a_{i p q} \sum_{n=0}^{\infty} \int_{0}^{t} A_{i 0, p, q} d t, \\
& x_{i 2}=\sum_{j=1}^{2} a_{i j} \int_{0}^{t} x_{i 1} d t+\sum_{p=1}^{2} \sum_{q=1}^{2} a_{i p q} \sum_{n=0}^{\infty} \int_{0}^{t} A_{i 1, p, q} d t, \\
& \vdots \\
& x_{i, n+1}=\sum_{j=1}^{2} a_{i j} \int_{0}^{t} x_{i n} d t+\sum_{p=1}^{2} \sum_{q=1}^{2} a_{i p q} \sum_{n=0}^{\infty} \int_{0}^{t} A_{i n, p, q} d t .
\end{aligned}
$$

Now by using only the nonlinear terms of the input functions in Algorithm bellow

\section{Algorithm (Computing Adomian Polynomials)[4]}

Input: the system

$$
\begin{array}{cc}
G_{1}=G\left(u_{1}, u_{2}, \ldots, u_{n}\right) \\
G_{2}=G\left(u_{1}, u_{2}, \ldots, u_{n}\right) \\
\vdots & \vdots \\
G_{k}= & G\left(u_{1}, u_{2}, \ldots, u_{n}\right)
\end{array}
$$

set $n=N, m=M, k=K$; the input of Adomian Polynomials needed.

Output: Aji; the Adomian Polynomials

Step 1: set $i=1$ 
Step 2: while $i \leq n$ we have:

$$
u_{i}(\lambda)=\sum_{b=0}^{m} u_{i b} \lambda^{b}
$$

Step 3: set $j=1$

Step 4: while $j \leq n$ do steps (5) and (6)

Step 5: $G_{i}(\lambda)=G_{i}\left(u_{j}(t)=u_{j}(\lambda)\right)$

Step 6: $G_{i}=G_{i}(\lambda)$

Step 7: $s_{i}=$ expansion of $G_{i}(\lambda)$ w.r.t. $\lambda$

$$
f t_{i}=s_{i}(\lambda)
$$

Step 8: while $j \leq k$ and while $j \leq m$

$$
A_{j i}=\frac{\partial^{i}}{\partial \lambda^{i}}\left(f t_{j}\right)(0) /(i) !=D^{i}\left(f t_{j}\right)(0) /(i) !
$$

Step 9: output Aji (the Adomian Polynomials)

Step 10: end.

From equation (10) we compute the Adomian Polynomials as follows:

$$
\begin{aligned}
& A_{10, p, q}=0 \\
& A_{11, p, q}=0 \\
& A_{12, p, q}=0 \\
& A_{13, p, q}=0 \\
& A_{14, p, q}=0
\end{aligned}
$$

and

$$
\begin{aligned}
A_{20, p, q}= & -\mu\left(x_{10}^{2} x_{20}\right) \\
A_{21, p, q}= & -D(\mu)\left(x_{10}^{2} x_{20}\right)\left(2 x_{10} x_{20} x_{11}+x_{10}^{2} x_{21}\right) \\
A_{22, p, q} & =-\frac{1}{2} D^{(2)}(\mu)\left(x_{10}^{2} x_{20}\right)\left(2 x_{10} x_{20} x_{11}+x_{10}^{2} x_{21}\right)^{2} \\
& -\frac{1}{2} D(\mu)\left(x_{10}^{2} x_{20}\right)\left(2 x_{11}^{2} x_{20}+4 x_{10} x_{21} x_{11}+4 x_{10} x_{20} x_{12}+2 x_{10}^{2} x_{22}\right) \\
A_{23, p, q}= & -\frac{1}{6} D^{(3)}(\mu)\left(x_{10}^{2} x_{20}\right)\left(2 x_{10} x_{20} x_{11}+x_{10}^{2} x_{21}\right)^{3} \\
& -\frac{1}{2} D^{(2)}(\mu)\left(x_{10}^{2} x_{20}\right)\left(2 x_{10} x_{20} x_{11}+x_{10}^{2} x_{21}\right)\left(2 x_{11}^{2} x_{20}+4 x_{10} x_{21} x_{11}+4 x_{10} x_{20} x_{12}\right. \\
& \left.+2 x_{10}^{2} x_{22}\right)--\frac{1}{6} D(\mu)\left(x_{10}^{2} x_{20}\right)\left(12 x_{11} x_{20} x_{12}+6 x_{11}^{2} x_{21}+12 x_{10} x_{22} x_{11}+\right. \\
& \left.+12 x_{10} x_{21} x_{12}+12 x_{10} x_{20} x_{13}+6 x_{10}^{2} x_{23}\right)
\end{aligned}
$$




$$
\begin{aligned}
A_{24, p, q} & =-\frac{1}{24} D^{(4)}(\mu)\left(x_{10}^{2} x_{20}\right)\left(2 x_{10} x_{20} x_{11}+x_{10}^{2} x_{21}\right)^{4} \\
& -\frac{1}{4} D^{(3)}(\mu)\left(x_{10}^{2} x_{20}\right)\left(2 x_{10} x_{20} x_{11}+x_{10}^{2} x_{21}\right)^{2}\left(2 x_{11}^{2} x_{20}+4 x_{10} x_{21} x_{11}\right. \\
& \left.+4 x_{10} x_{20} x_{12}+2 x_{10}^{2} x_{22}\right)-\frac{1}{8} D^{(2)}(\mu)\left(x_{10}^{2} x_{20}\right)\left(2 x_{11}^{2} x_{20}+4 x_{10} x_{21} x_{11}\right. \\
& \left.+4 x_{10} x_{20} x_{12}+2 x_{10}^{2} x_{22}\right)-\frac{1}{6} D^{(2)}(\mu)\left(x_{10}^{2} x_{20}\right)\left(2 x_{10} x_{20} x_{11}+x_{10}^{2} x_{21}\right)\left(12 x_{10} x_{20} x_{12}\right. \\
& \left.+6 x_{11}^{2} x_{21}+12 x_{10} x_{22} x_{11}+12 x_{10} x_{21} x_{12}+12 x_{10} x_{20} x_{13}+6 x_{10}^{2} x_{23}\right) \\
& -\frac{1}{24} D(\mu)\left(x_{10}^{2} x_{20}\right)\left(24 x_{12}^{2} x_{20}+48 x_{11} x_{21} x_{12}+48 x_{11} x_{20} x_{13}+24 x_{11}^{2} x_{22}\right. \\
& \left.+48 x_{10} x_{23} x_{11}+48 x_{10} x_{22} x_{12}+48 x_{10} x_{21} x_{13}+48 x_{10} x_{20} x_{14}+24 x_{10}^{2} x_{24}\right)
\end{aligned}
$$

and so on

Since $A_{1 n, p, q}=0, \forall n=0,1,2, \ldots$

$\therefore \quad \int_{0}^{t} A_{1 n, p, q}=0 \quad, \forall n=0,1,2, \ldots \quad$ and hence

$x_{10}=x_{1}(t=0)$

$x_{11}=\sum_{j=1}^{2} a_{1 j} \int_{0}^{t} x_{10} d t$

$x_{12}=\sum_{j=1}^{2} a_{1 j} \int_{0}^{t} x_{11} d t$

$x_{1, n+1}=\sum_{j=1}^{2} a_{1 j} \int_{0}^{t} x_{1 n} d t$

also

$$
\begin{aligned}
\int_{0}^{t} A_{20, p, q} d t & =-\mu\left(x_{10}^{2} x_{20}\right) t \\
\int_{0}^{t} A_{21, p, q} d t & =\frac{1}{2}\left(-D(\mu)\left(x_{10}^{2} x_{20}\right)\left(2 x_{10} x_{20} x_{11}+x_{10}^{2} x_{21}\right)\right) t^{2} \\
\int_{0}^{t} A_{22, p, q} d t & =\frac{1}{3}\left(-\frac{1}{2} D^{(2)}(\mu)\left(x_{10}^{2} x_{20}\right)\left(2 x_{10} x_{20} x_{11}+x_{10}^{2} x_{21}\right)^{2}\right. \\
- & \left.\frac{1}{2} D(\mu)\left(x_{10}^{2} x_{20}\right)\left(2 x_{11}^{2} x_{20}+4 x_{10} x_{21} x_{11}+4 x_{10} x_{20} x_{12}+2 x_{10}^{2} x_{22}\right)\right) t^{3}
\end{aligned}
$$




$$
\begin{aligned}
\int_{0}^{t} A_{23, p, q} d t & =\frac{1}{4}\left(-\frac{1}{6} D^{(3)}(\mu)\left(x_{10}^{2} x_{20}\right)\left(2 x_{10} x_{20} x_{11}+x_{10}^{2} x_{21}\right)^{3}\right. \\
& -\frac{1}{2} D^{(2)}(\mu)\left(x_{10}^{2} x_{20}\right)\left(2 x_{10} x_{20} x_{11}+x_{10}^{2} x_{21}\right)\left(2 x_{11}^{2} x_{20}+4 x_{10} x_{21} x_{11}\right. \\
& \left.+4 x_{10} x_{20} x_{12}+2 x_{10}^{2} x_{22}\right)-\frac{1}{6} D(\mu)\left(x_{10}^{2} x_{20}\right)\left(12 x_{11} x_{20} x_{12}+6 x_{11}^{2} x_{21}\right. \\
& \left.\left.+12 x_{10} x_{22} x_{11}+12 x_{10} x_{21} x_{12}+12 x_{10} x_{20} x_{13}+6 x_{10}^{2} x_{23}\right)\right) t^{4}
\end{aligned}
$$

hence we obtain

$$
\begin{aligned}
x_{20}= & x_{2}(t=0) \\
x_{21}= & \sum_{j=1}^{2} a_{2 j} \int_{0}^{t} x_{20} d t+\sum_{p=1}^{2} \sum_{q=1}^{2} a_{2 p q}\left(-\mu\left(x_{10}^{2} x_{20}\right) t\right) \\
x_{22}= & \sum_{j=1}^{2} a_{2 j} \int_{0}^{t} x_{21} d t+\sum_{p=1}^{2} \sum_{q=1}^{2} a_{2 p q}\left(\frac{1}{2}\left(-D(\mu)\left(x_{10}^{2} x_{20}\right)\left(2 x_{10} x_{20} x_{11}+x_{10}^{2} x_{21}\right)\right) t^{2}\right) \\
x_{23}= & \sum_{j=1}^{2} a_{2 j} \int_{0}^{t} x_{22} d t+\sum_{p=1}^{2} \sum_{q=1}^{2} a_{2 p q}\left(\frac { 1 } { 3 } \left(-\frac{1}{2} D^{(2)}(\mu)\left(x_{10}^{2} x_{20}\right)\left(2 x_{10} x_{20} x_{11}+x_{10}^{2} x_{21}\right)^{2}\right.\right. \\
& \left.\left.-\frac{1}{2} D(\mu)\left(x_{10}^{2} x_{20}\right)\left(2 x_{11}^{2} x_{20}+4 x_{10} x_{21} x_{11}+4 x_{10} x_{20} x_{12}+2 x_{10}^{2} x_{22}\right)\right) t^{3}\right) \\
x_{24}= & \sum_{j=1}^{2} a_{2 j} \int_{0}^{t} x_{23} d t+\sum_{p=1}^{2} \sum_{q=1}^{2} a_{2 p q} \frac{1}{4}\left(-\frac{1}{6} D^{(3)}(\mu)\left(x_{10}^{2} x_{20}\right)\left(2 x_{10} x_{20} x_{11}+x_{10}^{2} x_{21}\right)^{3}\right. \\
& \quad-\frac{1}{2} D^{(2)}(\mu)\left(x_{10}^{2} x_{20}\right)\left(2 x_{10} x_{20} x_{11}+x_{10}^{2} x_{21}\right)\left(2 x_{11}^{2} x_{20}+4 x_{10} x_{21} x_{11}\right. \\
& \left.+4 x_{10} x_{20} x_{12}+2 x_{10}^{2} x_{22}\right)-\frac{1}{6} D(\mu)\left(x_{10}^{2} x_{20}\right)\left(12 x_{11} x_{20} x_{12}+6 x_{11}^{2} x_{21}\right. \\
& \left.\left.\left.+12 x_{10} x_{22} x_{11}+12 x_{10} x_{21} x_{12}+12 x_{10} x_{20} x_{13}+6 x_{10}^{2} x_{23}\right)\right) t^{4}\right)
\end{aligned}
$$

and so on

Upon calculating the polynomials (10) and integrating, one then has for all $t \geq 0$ :

$$
x_{i}(t)=\sum_{n=0}^{\infty} d_{i n} \frac{t^{n}}{n !} \quad, \quad i=1,2
$$

Where the coefficients $d_{i n}$ are given by

$d_{i 0}=x_{i}(t)$,

$d_{i n}=\sum_{j=1}^{2} a_{i j} d_{j(n-1)}+(n-1) ! \sum_{p=1}^{2} \sum_{q=1}^{2} \sum_{k=0}^{n} a_{i p q} \frac{d_{q k}^{2}}{k !} \frac{d_{p(n-k-1)}}{k !(n-k-1) !} ; n \geq 1$

Hence from (27)-(29), the explicit solution to the autonomous Van der Pol system (2) is: 


$$
\begin{array}{ll}
x=\sum_{n=0}^{\infty} a_{n} \frac{t^{n}}{n !} & , n \geq 1 \\
y=\sum_{n=0}^{\infty} b_{n} \frac{t^{n}}{n !} & , n \geq 1
\end{array}
$$

Where the coefficients are given by the recurrence relations

$a_{0}=x(t=0), b_{0}=y(t=0)$,

$a_{1}=y_{0}$

$b_{1}=-x_{0}-\mu\left(x_{0}^{2}-1\right) y_{0}$

$$
a_{n}=b_{n-1} \quad, n \geq 1
$$

$b_{n}=-a_{n-1}+\mu b_{n-1}-\mu(n-1) ! \sum_{k=0}^{n-1} \frac{a_{k}^{2} b_{n-k-1}}{k !(n-k-1) !}, n \geq 1$

Again, Recall [4], we have been considered the numerical results of this method. This results show the efficient and powerful of Multistage Adomian Decomposition method when compared with RK4 and classical ADM for finding the solution of the system (2).

Case 1: $\mu=2$, and the initial conditions $x(0)=x_{0}=0.1$ and $y(0)=y_{0}=1$ Case 2: $\mu=5$, and the initial conditions $x(0)=x_{0}=0.1$ and $y(0)=y_{0}=1$ Case 3: $\mu=10$, and the initial conditions $x(0)=x_{0}=0.1$ and $y(0)=y_{0}=1$

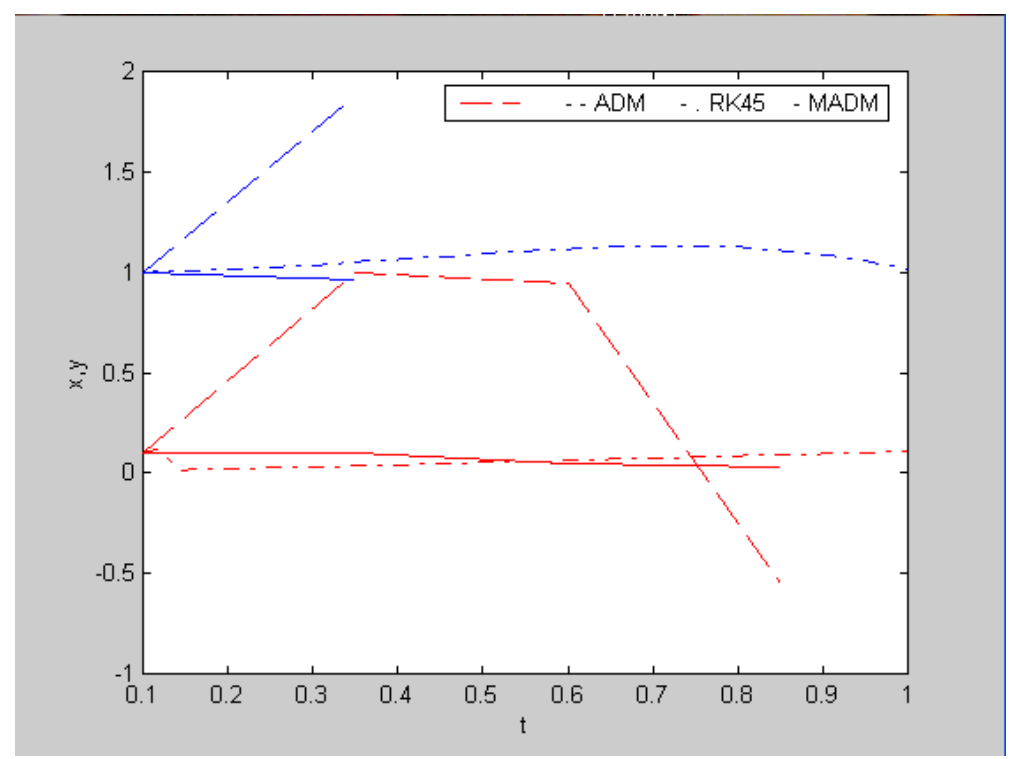

Fig. (1): Comparison of the solution of system (2) $x$ and $y$ at time $t$ for case 1 using 4-term classical ADM, 4-term MADM and RK45. 


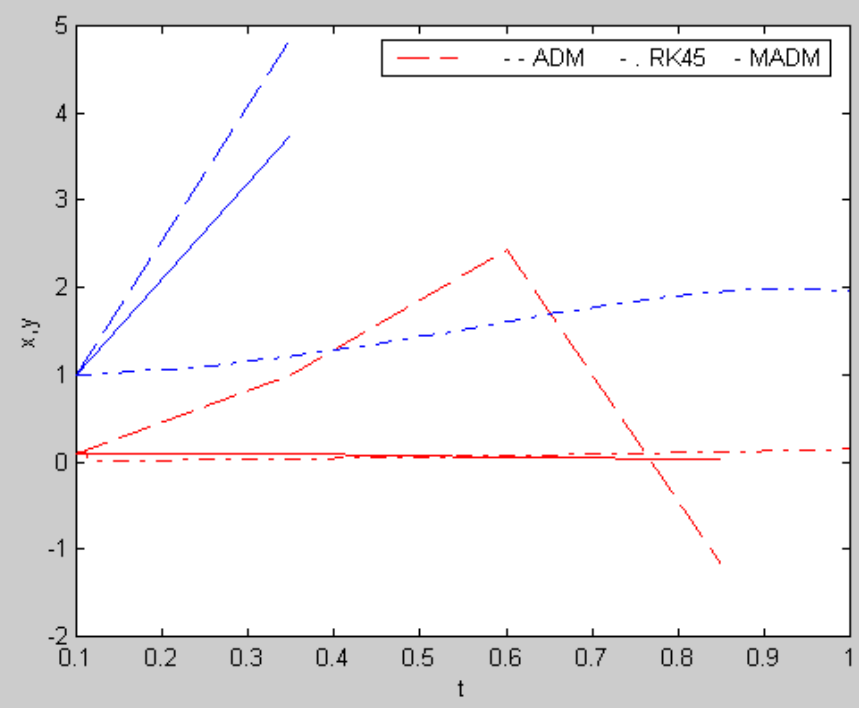

Fig. (2): Comparison of the solution of system (2) $x$ and $y$ at time $t$ for case 2 using 4-term classical ADM, 4-term MADM and RK45.

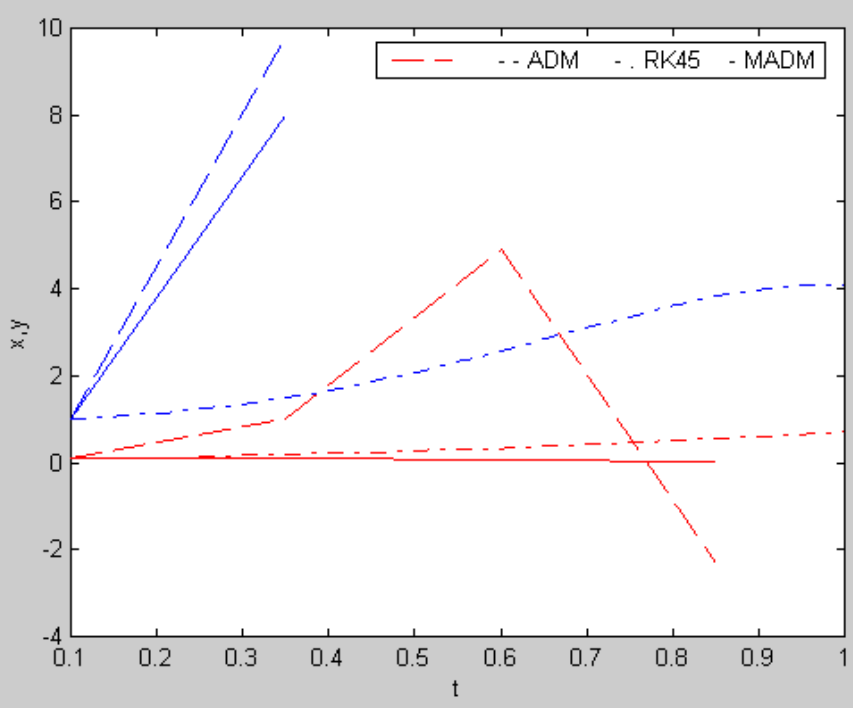

Fig. (3) Comparison of the solution of system (2) $x$ and $y$ at time $t$ for case 3 using 4-term classical ADM, 4-term MADM and RK45.

As depicted in Fig. (1), Fig.(2) and Fig. (3), these 4-term ADM solutions are not accurate enough. However, our 4-term MADM solutions agree very well with the RK45 solutions.

Now in order to obtain the convergence of the MADM, we have to derive the Homotopy analysis method for the system: 


\section{The Derivation of the Homotopy Analysis method for system}

(2)

Consider a nonlinear differential operator $\tilde{R}_{i 2}$, let $h \neq 0$ and $\lambda$ be complex numbers and $A_{i}(\lambda)$ and $B_{i}(\lambda), i=1,2$ be complex functions analytic in the region $|\lambda| \leq 1$, satisfying

$$
A_{i}(0)=B_{i}(0)=0 \quad, \quad A_{i}(1)=B_{i}(1)=1 \quad, \quad i=1,2
$$

respectively. Besides, let

$$
A_{i}(\lambda)=\sum_{k=1}^{\infty} \alpha_{i 1, k} \lambda^{k} \quad, \quad B_{i}(\lambda)=\sum_{k=1}^{\infty} \beta_{i 1, k} \lambda^{k}
$$

denote the Maclaurin's series of $A_{i}(\lambda)$ and $B_{i}(\lambda), i=1,2$ respectively. Because $A_{i}(\lambda)$ and $B_{i}(\lambda), i=1,2$ are analytic in the region $|\lambda| \leq 1$, therefore we have

$$
A_{i}(1)=\sum_{k=1}^{\infty} \alpha_{i 1, k}=1, \quad B_{i}(1)=\sum_{k=1}^{\infty} \beta_{i 1, k}=1, \quad i=1,2
$$

The above defined complex functions $A_{i}(\lambda)$ and $B_{i}(\lambda), i=1,2$ are called the embedding and $\lambda$ is the embedding parameter.

Consider

$$
\widetilde{R}_{i 2}\left(x_{i}(t)\right)=0, \quad t \geq 0, \quad i=1,2
$$

Where $\tilde{R}_{i 2}$ is a differential operator, $x_{i}(t)$ is a solution defined for $t \geq 0$.

To solve Equation (38) using Homotopy analysis method we construct the equation

$$
\left[1-B_{i}(\lambda)\right]\left\{£\left[\tilde{x}_{i}(t, \lambda)-\left(x_{i}(t=0)+\sum_{j=1}^{2} \sum_{n=0}^{\infty} a_{i j} \int_{0}^{t} x_{i n} d t\right)\right]\right\}=h A_{i}(\lambda) \tilde{R}_{i 2}\left[\tilde{x}_{i}(t, \lambda)\right]
$$

Where $£$ is a properly selected auxiliary linear operator satisfying

$$
\mathfrak{£}(0)=0
$$

and $h \neq 0$ is an auxiliary parameter, $x_{i}(t=0)$ is an initial approximation and $\sum_{j=1}^{2} \sum_{n=0}^{\infty} a_{i j} \int_{0}^{t} x_{i n} d t$ is the solution of linear term.

Using the facts that $A_{i}(0)=0$ and $B_{i}(0)=0, i=1,2$ Eq. (39) gives

$$
£\left[\tilde{x}_{i}(t, 0)-\left(x_{i}(t=0)+\sum_{j=1}^{2} \sum_{n=0}^{\infty} a_{i n} \int_{0}^{t} x_{i n} d t\right)\right]=0, \quad i=1,2
$$

or equivalently

$$
\tilde{x}_{i}(t, 0)=x_{i}(t=0)+\sum_{j=1}^{2} \sum_{n=0}^{\infty} a_{i j} \int_{0}^{t} x_{i n} d t
$$

Similarly, when $\lambda=1$, Eq. (39) is the same as Eq. (38) so that we have 
$\tilde{x}_{i}(t, 1)=x_{i}(t)$

Suppose that Eq. (38) has solution of nonlinear term $\tilde{x}_{i}(t, \lambda)$ that is $\int_{0}^{t} \widetilde{R}_{i 2}$ that converges for all $0 \leq \lambda \leq 1$ for properly selected $h, A_{i}(\lambda)$ and $B_{i}(\lambda), i=1,2$.

Suppose further that $\tilde{x}_{i}(t, \lambda)$ is infinitely differentiable at $\lambda=0$ that is

$$
\begin{aligned}
& \tilde{x}_{i 0}^{k}(t)=\left.\frac{d^{k} \tilde{x}_{i}(t, \lambda)}{d \lambda^{k}}\right|_{\lambda=0}, \quad k=0,1,2,3, \ldots \ldots . \\
& \Rightarrow \tilde{x}_{i 0}^{k}(t)=\frac{d^{k}}{d \lambda^{k}}\left(\sum_{p=1}^{2} \sum_{q=1}^{2} a_{i p q} \int_{0}^{t} \tilde{R}_{i 2}\left(x_{p}, x_{q}\right) d t\right), \quad i=1,2
\end{aligned}
$$

where $\tilde{R}_{i 2}(x, y)=x^{2} y$ exists for all $\mathrm{k}=0,1,2, \ldots$ Thus as $\lambda$ increases from 0 to 1 , the solution $\tilde{x}_{i}(t, \lambda)$ of the nonlinear term of Eq. (39), i.e. $\int_{0}^{t} \tilde{R}_{i 2}$, varies continuously from $\tilde{x}_{i}(t, 0)$ to the solution $x_{i}(t)$ of the original Eq. (38). Clearly, Eq. (41) and Eq. (42) gives an indirect relation between $\tilde{x}_{i}(t, 0)$ and the general solution $x_{i}(t)$.

The homotopy analysis method depends on finding a direct relationship between the two solutions which can be described as follows:

Consider the Maclaurin's series of $\tilde{x}_{i}(t, \lambda)$ about $\lambda=0$

$$
\begin{aligned}
& \tilde{x}_{i}(t, \lambda)=\tilde{x}_{i}(t, 0)+\sum_{k=1}^{\infty}\left(\left.\frac{d^{k} \tilde{x}_{i}(t, \lambda)}{d \lambda^{k}}\right|_{\lambda=0}\right) \frac{\lambda^{k}}{k !} \\
& \tilde{x}_{i}(t, \lambda)=\tilde{x}_{i}(t, 0)+\sum_{p=1}^{2} \sum_{q=1}^{2} a_{i p q} \sum_{k=1}^{\infty}\left(\left.\int_{0}^{t} \frac{d^{k} \widetilde{R}_{i 2}\left(x_{p}, x_{q}, \lambda\right)}{d \lambda^{k}}\right|_{\lambda=0} d t\right) \frac{\lambda^{k}}{k !} .
\end{aligned}
$$

Assuming that the series above converges at $\lambda=1$, we have by Eqs. (41) and (42) the relationship:

$$
x_{i}(t)=x_{i}(t=0)+\sum_{j=1}^{2} \sum_{n=0}^{\infty} a_{i j} \int_{0}^{t} x_{i n} d t+\sum_{n=1}^{\infty} x_{i n}(t)
$$

where

$$
x_{i n}(t)=\frac{\tilde{x}_{i 0}^{n}(t)}{n !}=\frac{\left.\frac{d^{n} \tilde{x}_{i}(t, \lambda)}{d \lambda^{n}}\right|_{\lambda=0}}{n !}=\left.\sum_{p=1}^{2} \sum_{q=1}^{2} a_{i p q} \int_{0}^{t} \frac{1}{n !} \frac{d^{n} \tilde{\mathrm{R}}_{\mathrm{i} 2}\left(x_{p}, x_{q}, \lambda\right)}{d \lambda^{n}}\right|_{\lambda=0}, n \geq 1
$$

To derive the governing equation of $x_{i n}(t)$, we differentiate Eq. (39) $\mathrm{n}$ times w. r.t. $\lambda$ we get 


$$
\begin{gathered}
\sum_{k=0}^{n}\left(\begin{array}{l}
n \\
k
\end{array}\right) \frac{d^{k}\left[1-B_{i}(\lambda)\right]}{d \lambda^{k}} \frac{d^{(n-k)}}{d \lambda^{(n-k)}}\left\{£\left[\tilde{x}_{i}(t, \lambda)-\left(x_{i}(t=0)+\sum_{j=0}^{2} \sum_{n=0}^{\infty} a_{i j} \int_{0}^{t} x_{i n} d t\right)\right]\right\} \\
=h \sum_{k=0}^{n}\left(\begin{array}{l}
n \\
k
\end{array}\right) \frac{d^{k} A_{i}(\lambda)}{d \lambda} \frac{d^{(n-k)} \tilde{\mathrm{R}}_{i 2}\left[\tilde{x}_{\mathrm{i}}(t, \lambda)\right]}{d \lambda^{(n-k)}}
\end{gathered}
$$

Further dividing Eq. (47) by $\mathrm{n}$ ! and then setting $\lambda=0$, we have the so called nth-order deformation equations

$$
\begin{aligned}
£\left[\sum_{p=1}^{2} \sum_{q=1}^{2} a_{i p q}\right. & \left.\int_{0}^{t} \frac{1}{n !} \frac{d^{n} \tilde{R}_{i 2}\left(x_{p}, x_{q}, \lambda\right)}{d \lambda^{n}}\right|_{\lambda=0} d t- \\
& \left.\quad-\left.\sum_{k=1}^{n-1} \beta_{i 1, k} \sum_{p=1}^{2} \sum_{q=1}^{2} a_{i p q} \int_{0}^{t} \frac{1}{(n-k) !} \frac{d^{(n-k)} \tilde{R}_{i 2}\left(x_{p}, x_{q}, \lambda\right)}{d \lambda^{(n-k)}}\right|_{\lambda=0} d t\right]=R_{i n}(t)
\end{aligned}
$$

where $R_{\text {in }}(t)$ in fact depends on the previous calculated values of

$$
x_{i}(t=0)+\sum_{j=1}^{2} \sum_{n=0}^{\infty} a_{i j} \int_{0}^{t} x_{i n} d t, \quad i=1,2
$$

are given by

$$
R_{i n}(t)=h \sum_{k=1}^{n} \alpha_{i 1, k} h_{i, n-k}(t)
$$

and $h_{i n}(t)$ are the homotopy polynomials given by

$$
h_{i n}(t)=\left.\frac{1}{n !} \frac{d^{n} \tilde{R}_{i 2}\left[\tilde{x}_{\mathrm{i}}(t, \lambda)\right]}{d \lambda^{n}}\right|_{\lambda=0} \quad, i=1,2
$$

We emphasize that Homotopy Analysis Method provides us with the large flexibility to select the nonzero auxiliary parameters $h$, the embebbing functions $A_{i}(\lambda)$ and $B_{i}(\lambda), i=1,2$ and the auxiliary linear operator $£$.

Now by comparing Eq. (50) with the Eq. (10) we conclude that Homotopy polynomials will be reduced to the Multistage Adomian decomposition polynomials and when $h=-1$, we conclude that $A_{\text {in }}(t)=h_{\text {in }}(t)$.

\section{The Convergence of MADM for system (2) using HAM}

In this section the question of convergence of MADM will be addressed. 


\section{Theorem 1.}

If the series

$$
x_{i}(t=0)+\sum_{j=1}^{2} \sum_{n=0}^{\infty} a_{i j} \int_{0}^{t} x_{i n} d t+\sum_{n=1}^{\infty} x_{i n}(t) d t
$$

where

$$
x_{i n}(t)=\frac{\tilde{x}_{i 0}(t)}{n !}=\frac{\left.\frac{d^{n} \tilde{x}_{i}(t, \lambda)}{d \lambda^{n}}\right|_{\lambda=0}}{n !}=\left.\sum_{p=1}^{2} \sum_{q=1}^{2} a_{i p q} \int_{0}^{t} \frac{1}{n !} \frac{d^{n} \tilde{\mathrm{R}}_{\mathrm{i} 2}\left(x_{p}, x_{q}, \lambda\right)}{d \lambda^{n}}\right|_{\lambda=0} d t, \quad n \geq 1
$$

is convergent, it must be a solution of system (2).

Proof. By Eq. (48) we have

$$
\begin{aligned}
& \sum_{n=1}^{\infty} R_{i n}(t)=\sum_{n=1}^{\infty} £\left[\left.\sum_{p=1}^{2} \sum_{q=1}^{2} a_{i p q} \int_{0}^{t} \frac{1}{n !} \frac{d^{n} \tilde{\mathrm{R}}_{\mathrm{i} 2}\left(x_{p}, x_{q}, \lambda\right)}{d \lambda^{n}}\right|_{\lambda=0} d t-\right. \\
& =£\left[\left.\left.\sum_{p=1}^{2} \sum_{q=1}^{2} a_{i p q} \sum_{n=1}^{\infty} \int_{0}^{t} \frac{1}{n !} \frac{d^{n} \tilde{\mathrm{R}}_{\mathrm{i} 2}\left(x_{p}, x_{q}, \lambda\right)}{d \lambda^{n}}\right|_{\lambda=0} ^{n-1} \beta_{i 1, k} \sum_{p=1}^{2} \sum_{q=1}^{2} a_{i p q} \int_{0}^{t} \frac{1}{(n-k) !} \frac{d^{(n-k)} \tilde{\mathrm{R}}_{\mathrm{i} 2}\left(x_{p}, x_{q}, \lambda\right)}{d \lambda^{(n-k)}}\right|_{\lambda=0} d t\right] \\
& =£\left[\left.\left.\sum_{p=1}^{2} \sum_{q=1}^{2} a_{i p q} \sum_{n=1}^{\infty} \int_{0}^{t} \frac{1}{n !} \frac{d^{n} \tilde{\mathrm{R}}_{\mathrm{i} 2}\left(x_{p}, x_{q}, \lambda\right)}{d \lambda^{n}}\right|_{\lambda=0} ^{\infty} \beta_{i 1, k}^{2} \sum_{p=1}^{2} \sum_{q=1}^{2} a_{i p q} \sum_{n=k+1}^{n-1} \int_{0}^{t} \frac{1}{(n-k) !} \frac{d^{(n-k)} \tilde{\mathrm{R}}_{\mathrm{i} 2}\left(x_{p}, x_{q}, \lambda\right)}{d \lambda^{(n-k)}}\right|_{\lambda=0} d t\right] \\
& \sum_{n=1}^{\infty} R_{i n}(t)=£\left[\left(1-\sum_{k=1}^{\infty} \beta_{i 1, k}\right)\left\{\left.\sum_{p=1}^{2} \sum_{p=1}^{2} a_{i p q} \sum_{n=1}^{\infty} \int_{0}^{t} \frac{1}{n !} \frac{d^{n} \tilde{\mathrm{R}}_{\mathrm{i} 2}\left(x_{p}, x_{q}, \lambda\right)}{d \lambda^{n}} \sum_{\lambda=0}^{2} \sum_{q=1}^{2} a_{i p q} \sum_{n=1}^{\infty} \int_{0}^{t} \frac{1}{(n-k) !} \frac{d^{n} \tilde{\mathrm{R}}_{\mathrm{i} 2}\left(x_{p}, x_{q}, \lambda\right)}{d \lambda^{n}}\right|_{\lambda=0} d t\right]\right.
\end{aligned}
$$

Recall that $\beta_{i}(\lambda)=\lambda, \quad i=1,2$ therefore

$$
\beta_{i 1, k}=\left\{\begin{array}{ccc}
0 & \text { when } & k=0 \\
1 & \text { when } & k=1 \\
0 & \text { when } & k>1
\end{array}\right\}
$$

Which given by Eq. (42)

$\sum_{n=1}^{\infty} R_{i n}(t)=0$

On the other hand by Eq. (49) and Eq. (50) that: 


$$
\begin{aligned}
\sum_{n=1}^{\infty} R_{i n}(t) & =\sum_{n=1}^{\infty} h \sum_{k=1}^{n} \alpha_{i 1, k} h_{i,(n-k)}(t)=h \sum_{n=1}^{\infty} \alpha_{i 1, k} \sum_{n=k}^{\infty} h_{i,(n-k)}(t)=h \sum_{n=1}^{\infty} \alpha_{i 1, k} \sum_{n=0}^{\infty} h_{i, n}(t) \\
= & \left.h \sum_{n=1}^{\infty} \alpha_{i 1, k} \sum_{n=0}^{\infty} \frac{1}{n !} \frac{d^{n} \tilde{R}_{i 2}\left[\tilde{x}_{i}(t, \lambda)\right]}{d \lambda^{n}}\right|_{\lambda=0}
\end{aligned}
$$

Again, $A_{i}(\lambda)=\lambda, i=1,2$, therefore

$$
\alpha_{i 1, k}=\left\{\begin{array}{lll}
0 & \text { when } & k=0 \\
1 & \text { when } & k=1 \\
0 & \text { when } & k>1
\end{array}\right\}
$$

Therefore, $\sum_{k=1}^{\infty} \alpha_{i 1, k}=1$, thus the above expression becomes

$$
\sum_{n=1}^{\infty} R_{i n}(t)=h \sum_{n=1}^{\infty} h_{i, n}(t)=\left.h \sum_{n=0}^{\infty} \frac{1}{n !} \frac{d^{n} \tilde{\mathrm{R}}_{i 2}\left[x_{i}(t, \lambda)\right]}{d \lambda^{n}}\right|_{\lambda=0}
$$

Note that $h=-1$, therefore, By Eqs. (53) and (54) we have

$$
\left.\sum_{n=0}^{\infty} \frac{1}{n !} \frac{d^{n} \tilde{R}_{i 2}\left[\tilde{x}_{i}(t, \lambda)\right]}{d \lambda^{n}}\right|_{\lambda=0}=0
$$

In addition, $\tilde{x}_{i}(t, \lambda)$ is not a solution of equation (2) in general when $\lambda \neq 1$. Now define $\Delta(t, \lambda)=R_{i 2}\left[\tilde{x}_{i}(t, \lambda)\right]-R_{i 2}\left[x_{i}(t)\right]=R_{i 2}\left[\tilde{x}_{i}(t, \lambda)\right]$, as a residual error of system (2) then The Maclaurin's series of this residual about $\lambda=0$ is

$$
\sum_{n=0}^{\infty} \frac{d^{n} \Delta(t, \lambda)}{d \lambda^{n}} \frac{\lambda^{n}}{n !}=\left.\sum_{n=0}^{\infty} \frac{d^{n} \mathrm{R}_{i 2}\left[\tilde{x}_{i}(t, \lambda)\right]}{d \lambda^{n}}\right|_{\lambda=0} \frac{\lambda^{n}}{n !}
$$

According to (50), the above Maclaurin's series converge at $\lambda=1$, say

$$
\Delta(t, 1)=R_{i 2}\left[\tilde{x}_{i}(t, 1)\right]=\left.\sum_{n=1}^{\infty} \frac{1}{n !} \frac{d^{n} R_{i 2}\left[\tilde{x}_{i}(t, \lambda)\right]}{d \lambda^{n}}\right|_{\lambda=0}=0
$$

Which means that

$$
\begin{aligned}
x_{i}(t)=\tilde{x}_{i}(t, 1)=x_{i}(t & =0)+\sum_{j=1}^{2} \sum_{n=0}^{\infty} a_{i j} \int_{0}^{t} x_{i n} d t+ \\
& +\left.\sum_{p=1}^{2} \sum_{q=1}^{2} a_{i p q} \int_{0}^{t} \frac{1}{n !} \frac{d^{n} \tilde{\mathrm{R}}_{\mathrm{i} 2}\left(x_{p}, x_{q}, \lambda\right)}{d \lambda^{n}}\right|_{\lambda=0} d t, \quad n \geq 1
\end{aligned}
$$

must be a solution of Eq.(2), which completes the proof.

To estimate whether the series converges or diverges, one can use the following theorem: 
Theorem 2. If the series in Eq. (51) converges then the following two sequences:

$$
\begin{aligned}
& \chi_{k}=\sum_{n=1}^{k} R_{i n}(t) \\
& v_{k}=\sum_{n=1}^{k} h_{i n}(t)
\end{aligned}
$$

Where $R_{i n}(t)$ and $h_{i n}(t)$ are defined by Eqs. (49) and (50) converges to zero.

Proof: The proof of this theorem is subsequent of Eqs. (53) and (55).

\section{Conclusions}

The derivation of the Homotopy Analysis method for system (2) has been applied in this paper. This method provides us with a convenient way of controlling the convergence of approximation series. We have been shown that if the series (51) converges then the two sequences $\chi_{k}=\sum_{n=1}^{k} R_{i n}(t)$ and $v_{k}=\sum_{n=1}^{k} h_{i n}(t)$ converge to zero.

\section{References}

1) Abdul aziz O., Noor N. F. M., Hashim I. and Noorani M. S. M., Further accuracy tests on Adomian decomposition method for chaotic systems. Chaos Solitons Fract 36: 1405-1411(2008).

2) Adomian G., "Nonlinear stochastic systems theory and application to physics". Dordrecht: Kluwer (1988).

3) Adomian G., "solving frontier problems of physics: The decomposition method". Boston: Kluwer Academic press (1994).

4) AL_ Bayati A. Y., AL_Sawoor A. J. and Samarji M. A., A Multistage Adomian Decomposition Method for solving the autonomous Van der Pol system, Australian Journal of Basic and Applied Sciences, 3(4): 4397-4407(2009).

5) Allan F., and Syam M., On the analytic solutions of the non homogeneous Blasius problem, J. Comput. App. Math. 182:36 371(2005).

6) Allan F., Derivation of the Adomian decomposition method using the homotopy analysis method, J. Comput. App. Math. 190: 614(2007).

7) Chowdhury M. S. H., Hashim I. and Mawa S., Solution of preypredator problem by Numeric-analytic technique. Communications in Nonlinear Science and Numerical Simulation 14: 10081012(2009). 
8) Ghosh S., Roy A. and Roy D., An adaptation of Adomian decomposition for numeric - analytic integration of strongly nonlinear and chaotic oscillations. Comput. Math. App. Mech Eng.196: 1133-1153(2007).

9) Guellal S., Grimalt P. and Y. Cherruault, Numerical study of Lorenz's equation by Adomian method. Comput. Math. Appl. 33: 25-9(1997).

10) Hashim I., Noorani M. S. M., Ahmad R., Bakar SA., Ismail ES. and Zakaria AM., Accuracy of the Adomian decomposition method applied to the Lorenz system. Chaos Solitons Fract.28:1149-1159(1997).

11) Liao S. J., An explicit totally analytic approximate solution for Blasius viscous flow problems, Int. J. Non-Linear Mech. 34: 759778(1999).

12) Liao S. J. and Chung K. F., Homotopy analysis of nonlinear progressive waves in deep water, J. Eng. Math. 45:105-116 (2003).

13) Liao S. J., An explicit analytic solution to the Thomas-Fermi equation, App. Math. Comput. 144: 433 - 444 (2003).

14) Liao S. J. and Compo A., Analytic solution of the temperature distribution in Blasuis viscous flow problem, J. Fluid Mech. 453: 411-425(2002).

15) Noorani M. S. M., Hashim I., Ahmad R., Bakar SA., Ismail ES. and Zakaria AM., Comparing numerical methods for the solutions of the Chen system. Chaos Solitons Fract.32:1296-1304(2007).

16) Olek S., An accurate solution to the multispecies Lotka-Volterra equations. SIAM Rev.36: 480-488(1994).

17) Ruan J. and $\mathrm{Lu}$ Z., A modified algorithm for Adomian decomposition method with applications to Lotka-Volterra systems. Math. And Computer Modelling, 46: 1214-1224 (2007).

18) Shawagfeh N. and Kaya D., Comparing numerical methods for the Solutions of systems of ordinary differential equations. Appl. Math. Lett.17: 323-8(2004).

19) Sonnad J. R. and Goudar C. T., Solution of the Haldane equation for substrate inhibition enzyme kinetics using the decomposition method. Math. Comput. Model. 40:573-82(2004).

20) Vadasz P. and Olek S., Convergence and accuracy of Adomian's decomposition method for the solution of Lorenz equation. Int $\mathbf{J}$ Heat Mass Transfer. 43:1715-1734(2000).

21) Van der Pol B., On Relaxation Oscillations. I, Phil. Mag. 2:978922 (1926). 